\title{
Study on the Content of Dietary Fiber in Barley Leaf Powder
}

\author{
Songling Cai ${ }^{1, *}$, Zhanghua Liao ${ }^{2}$ \\ ${ }^{1}$ Department of Food Quality and Safety, College of Management, Shanghai Sanda University, Shanghai, China \\ ${ }^{2}$ Department of Food Science and Engineering, College of Agriculture and Biology, Shanghai Jiaotong University, Shanghai, China
}

Email address:

caisongling598@126.com (Songling Cai),853252672@qq.com (Zhanghua Liao)

\section{To cite this article:}

Songling Cai, Zhanghua Liao. Study on the Content of Dietary Fiber in Barley Leaf Powder. Science Discovery.

Vol. 6, No. 4, 2018, pp. 243-248. doi: 10.11648/j.sd.20180604.13

Received: June 25, 2018; Accepted: July 13, 2018; Published: July 20, 2018

\begin{abstract}
Barley leaves are rich in dietary fiber, vitamins, proteins, minerals and other nutrients. They are the most popular functional foods in Hongkong, Japan, North America and Southeast Asia in recent years. The dietary fiber has become a hot subject for the research of the food industry with its physicochemical property and the prevention of high blood fat, diabetes, obesity and other physiological functions. Therefore, this paper aims to study the content of dietary fiber in barley leaf powder and determine the content of soluble dietary fiber and insoluble dietary fiber by enzymatic-gravimetric method using the total amount of Megazyme dietary fiber test kit. Samples of five kinds of common barley(Yannong No. 1, Subei 4, Subei 9, Siyin 3 and Dongxin 1) were prepared. The results were as follows: the content range of insoluble dietary fiber was $1.71 \%-6.69 \%$, the content range of soluble dietary fiber was $0.60 \%-2.26 \%$, and the range of RSD value was $0.62 \%-7.97 \%$. These experimental data and testing methods can provide reference for the scholars who study the nutritional efficacy of barley leaf powder, and further promote the market development of this kind of products in China.
\end{abstract}

Keywords: Barley Leaf Powder, Dietary Fiber, Soluble Dietary Fiber, Insoluble Dietary Fiber, Determine

\section{大麦若叶粉中的膳食纤维含量研究}

\author{
蔡松铃 ${ }^{*}$, 廖樟华 ${ }^{2}$ \\ 1管理学院, 食品质量与安全系, 上海杉达学院, 上海, 中国 \\ 2农业与生物学院, 食品科学与工程系, 上海交通大学, 上海, 中国
}

\section{邮箱}

caisongling598@126.com(蔡松铃),853252672@qq.com(廖樟华)

\begin{abstract}
摘要: 大麦若叶产品富含膳食纤维、维生素、蛋白质和矿物质等一系列营养素, 是近年来在香港、日本、北美、东南 亚等地比较风靡的功能性食品, 而膳食纤维因其特有的物理和化学性质以及预防高血脂、糖尿病、肥胖等生理功能, 成为食品界学者们研究的热门对象。故本文旨在研究大麦若叶粉中的膳食纤维含量, 利用Megazyme膳食纤维总量检测 试剂盒, 采用酶重量法测定样品中可溶性膳食纤维与不溶性膳食纤维的含量。样品选取五种常见大麦 (盐农啤 1 号、苏 啤4号、苏啤 9 号、西引 3 号和东辛 1 号), 经种植后制备而成, 检测结果为: 不可溶性膳食纤维含量范围为 $1.71 \%-6.69 \%$, 可溶性膳食纤维含量范围为 $0.60 \%-2.26 \%$, RSD值的变化范围为 $0.62 \%-7.97 \%$ 。这些实验数据和检测方法能为研究大麦 若叶粉营养功效的学者们提供参考依据, 从而进一步推动该类产品在中国的市场开拓。
\end{abstract}

关键词: 大麦若叶粉, 膳食纤维, 可溶性膳食纤维, 不溶性膳食纤维, 检测 


\section{1. 引言}

膳食纤维是指在小肠内不能被人体消化吸收的碳水 化合物聚合物, 化学组成包括纤维状碳水化合物 (纤维素)、 基质碳水化合物（果胶类物质、半纤维素）及填料类物质 (木质素) 三大类。它是一种极其重要的营养成分, 是继 人类所需 6 大营养素之后能够调节机体功能的第七大营养 素。根据膳食纤维的溶解性, 将其分为可溶性膳食纤维与 不可溶性膳食纤维, 两者对人体产生的作用也是各有差异 的。同时大麦若叶产品作为功能性食品, 因其具有减肥、 预防高血压、高血脂、糖尿病、脂肪肝等症状而备受人们 青睐。其制品已获得日本健康协会认定的健康食品标志, 美国也将麦绿素正式批准为食品增补剂。中国对大麦苗产 品研究较晚, 1994年徐新月博士正式将麦绿素概念从美国 引入中国。目前麦苗类保健食品不仅在日本、欧美等发达 国家流行，而且在我国香港、台湾及东南亚地区亦十分畅 销。在中国大陆, 麦苗产品的研究开发和市场前景十分看 好, 目前中国也有诸多企业开始自主研发大麦苗粉系列产 品, 如“大麦若叶”、“大麦苗提取汁粉”、“麦绿片”、“大麦 苗粉”等。

大麦苗是膳食纤维的良好来源, 但目前针对大麦苗粉 中可溶性膳食纤维和不可溶性膳食纤维含量测定的研究 甚少。美国谷物化学师协会 (AACC) 和美国分析化学家 协会 (AOAC) 早在 20 世纪 80 年代就制定了腼食纤维的相 关检测标准, 之后中国也制定了食品安全国家标准, 测定 食品中膳食纤维, 可见对于膳食纤维含量的研究显得尤为 重要。故本文采用通过AOAC和AACC认证的Megazyme 试剂盒测定方法, 选择长至 $20-30 \mathrm{~cm}$ 的大麦苗样品, 冷冻 干燥成大麦若叶粉, 检测其中可溶性与不溶性膳食纤维含 量, 为大麦若叶粉的营养功效和生理功能研究提供参考依 据, 对打开该类产品的中国市场具有深远的社会意义, 也 能进一步推动该类产品在食品中的应用。

\section{2. 大麦若叶粉的研究进展}

大麦(Hordeum vulgare), 属大麦属(Hordeum), 早熟禾 科(Poaceae), 大麦若叶是大麦生长到20-30公分的幼苗, 此时期的幼苗所含的各种营养素最为丰富, 其嫩苗富含叶 绿素、黄酮、维生素、矿物质等多种功能营养成分。[1] 大麦若叶粉是选用苗高 $20 \sim 30 \mathrm{~cm}$ 的新鲜大麦嫩茎叶,经割 青、篮选、清洗、杀青、干燥、粉碎等工序所制成, 水分 含量在 $4 \% \sim 5 \%$, 常温咜存期为 12 个月以上的粉状制品 [2]。 大麦若叶粉是具有多种营养保健功能的碱性食品, 在日韩、 美国、澳大利亚等地畅销[2], 深受大家推崇。虽然我国大 麦资源丰富, 很适合大规模生产，但是国内消费者对此类 功能性食品的关注度和接受度尚浅, 所以我国的学者和企 业一直在努力开发此类产品, 不断加强技术研究, 使其营 养价值和保健功能加以推广应用。

\section{1. 大麦若叶粉的营养价值}

大麦若叶粉营养丰富, 其主要营养成分含量都较高。 相关研究表明 [3], 大麦嫩苗是一种高蛋白食物, 蛋白质含 量高达 $28.2 \%$ 。经测定, 嫩苗中富含 18 种氨基酸, 尤其是必 需氨基酸种类占总氨基酸的 $39 \%[4]$ 。麦苗也含有丰富的天 然维生素 $\mathrm{C}$ 、维生素 $\mathrm{E}$ 、和维生素 $\mathrm{B}$ 。其中维生素 $\mathrm{C}$ 及维生素 E含量可以达到14.9、6.94mg $100 \mathrm{~g}$, 而维生素B1、维生素 B2、维生素B6的含量分别为 $52 、 244 、 175 \mu \mathrm{g} / 100 \mathrm{~g}[3]$ 。所 含人体必需的微量元素中含量最高的为 $\mathrm{K} 、 \mathrm{Ca} 、 \mathrm{Mg}, \mathrm{Ca}$ 含 量是牛奶的 4 倍, 其次是 $\mathrm{Fe} 、 \mathrm{Se} 、 \mathrm{Zn}$, 含量最低的是 $\mathrm{Cu}$ 和 $\mathrm{Mn}[5]$ 。大麦若叶粉的碱性度高达 66.4 , 被称为“碱性食品 之王”。当体内矿物质处于平衡状态时, 对维持人体健康有 利[6]。膳食纤维的含量甚至是普通青菜的 50 倍, 有“绿色血 液”之称的叶绿素提取量达 $13.622 \mathrm{mg} / \mathrm{g}[7]$, 此外, 大麦若叶 粉中含大量具有较强抗氧化活性的黄酮类化合物。

\section{2. 大麦若叶粉的加工工艺}

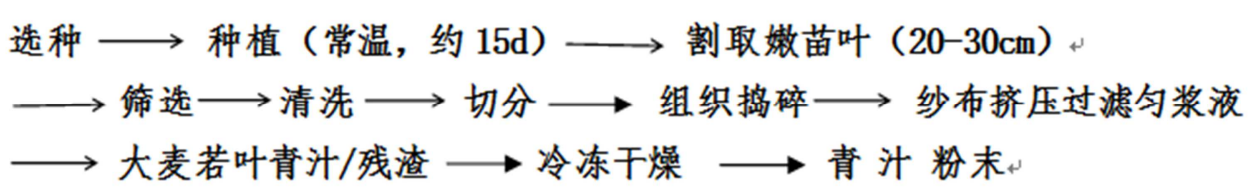

图1 大麦若叶粉的工艺流程图。

选取 5 种大麦品种种子 (品种信息见表 1 ) 为实验对象, 按照产品说明要求在室温下种植生长约 15 天后, 割取 $20-30 \mathrm{~cm}$ 高的大麦嫩苗, 去除枯黄的麦苗, 剔除杂草, 清洗去除泥沙杂质, 切成 $2 \sim 4 \mathrm{~cm}$ 左右的麦苗段, 放入搅拌机中进行组织 捣碎, 控制料液比例为 $1: 20$, 同时分三次加入去离子水, 每隔30秒组织摚拌 2 分钟, 捣碎结束后用 200 目纱布过滤挤压 捣碎匀浆液, 分别得到大麦残渣和大麦青汁液, 最后放入冷冻干燥机中冷冻干燥成粉（具体参数见表2）, 计算得出大 麦若叶粉得率为 $2.44-3.6 \%$ 。

表1 大麦样品信息表。

\begin{tabular}{lllll}
\hline 序号 & 大麦品种名 & 大麦鲜嫩叶重 $(\mathbf{g})$ & 青汁液粉末重 $(\mathbf{g})$ \\
\hline 1 & 盐农啤1号 & 564.6 & 19.3 & 3.42 \\
2 & 苏啤4号 & 417.4 & 10.2 & 2.44 \\
3 & 苏啤9号 & 550.3 & 19.8 & 3.6 \\
4 & 西引3号 & 420.3 & 14.2 & 3.38 \\
\hline
\end{tabular}




\begin{tabular}{lllll}
\hline 序号 & 大麦品种名 & 大麦鲜嫩叶重 $(\mathrm{g})$ & 青汁液粉末重 $(\mathrm{g})$ & 得率 $(\%)$ \\
\hline 5 & 东辛1号 & 605.3 & 20 & 3.3 \\
\hline
\end{tabular}

表2 立式冷冻干燥机参数表。

\begin{tabular}{llllll}
\hline 梯度 & 设定温度 $\left({ }^{\circ} \mathbf{C}\right)$ & 设定时间 & 梯度 & 设定温度 $\left({ }^{\circ} \mathbf{C}\right)$ & 设定时间 \\
\hline 1 & -20.0 & 99 时 & 6 & $5^{\circ} \mathrm{C}$ & 1 时 \\
2 & -15.0 & 1时 & 7 & $10^{\circ} \mathrm{C}$ & 1 时 \\
3 & -10.0 & 1时 & 8 & $25^{\circ} \mathrm{C}$ & 0 时 \\
4 & -5.0 & 2 时 & 9 & $20^{\circ} \mathrm{C}$ & 0 时 \\
5 & 0.0 & 2 时 & 10 & $25^{\circ} \mathrm{C}$ & 99 时 \\
\hline
\end{tabular}

备注: 以上大麦若叶粉加工工艺条件均在上海交通大学农业与生物学院谷物实验室内完成, 故不可完全等同于企业车间工艺操作, 仅供参考, 适用 于实验室内后续实验。

\section{3. 噮食纤维的定义}

20 世纪 50 年代, 膳食纤维(DF)的概念首次出现在科学 文献中, 该术语特指纤维素、半纤维素和木质素, 并指出 膳食纤维是一种不被人体消化酶所消化的植物成分的总 称 [8]。1999年美国谷物化学家协会(AACC)对膳食纤维作 出了如下定义:膳食纤维是指能抵抗人体小肠消化吸收的、 在人体大肠能部分或全部发酵的可食用的植物性成分, 是 碳水化合物及其相类似物质的总和, 包括多糖、寊糖、木 质素以及相关的植物物质。随着对膳食纤维的不断深入研 究, 目前, 膳食纤维已被许多国家定义为是长时间聚合度 (DP)大于 10 的抗性多糖[9]。根据膳食纤维来源的溶解性不 同，一般有两种分类：可溶性纤维(如果胶、瓜尔胶、葡 聚糖等胶类和糖类)和不溶性纤维(如麦麸、大豆壳等纤维 素、木质素和半纤维素)。膳食纤维因其特有的生理功能 和物化特性而被学者们广泛地研究与应用, 现已成为功能 性食品研究领域的热门方向。

\section{4. 膳食纤维的生理功能}

大量的研究证明, 膳食纤维的摄入可以减少如高血压、 高血脂、肥胖及糖尿病等许多疾病的患病风险, 同时有促 进肠道消化吸收、提高肠免疫、抗癌、润肠通便等功效 $[10]$ 。

\section{1. 调节血糖功能}

膳食纤维能有效预防糖尿病, 其含有的果胶可延长食 物在肠内的停留时间, 延缓淀粉等可消化糖类物质的消化 过程, 避免餐后血糖迅速增加, 从而维持血糖的平衡和稳 定。此外, 食用膳食纤维可增强机体对胰岛素的敏感性, 增高机体对葡萄糖耐受能力, 一定程度降低糖尿早期患者 对肤岛素的需求, 在提高肝脏中与糖分解代谢有关酶的活 性时, 肝细胞上增多的胰岛素受体数目增加了与胰岛素的 结合能力[11]。

\section{2. 降血脂功能}

膳食纤维降血脂作用的机理主要通过以下几种途径 来实现: (1)降低扩散速率, 抑制肠道内胆固醇的吸收; (2) 降低脂质代谢物的吸收率, 促进胆固醇转化为胆汁酸; (3) 阻碍胆汁酸重吸收进入肝肠循环, 抑制肝脏胆固醇的合成;
(4)促进胆固醇的排泄; (5)增加血浆胆固醇的清除这些途径 来实现的[12]。

\section{3. 提高肠道功能}

经消化吸收后的食物残渣到达结肠后被微生物发酵, 人体摄入膳食纤维后, 对在发酵过程中产生的有毒代谢物 具有吸附、鳌合作用, 从而减少其对肠壁的刺激, 膳食纤 维中的纤维素作为植物细胞壁的纤维成分, 为肠道细菌提 供能量底物, 刺激肠道有益细菌的增殖, 维持了肠道微生 态的平衡和稳定, 从而提高肠道的免疫功能[13]。

\section{4. 润肠通便}

水溶性膳食纤维在肠道内呈溶液状态, 有较好的持水 力,且易被肠道细菌酵解, 产生丁酸、丙酸、乙酸等短链脂 肪酸(SCFA), 这些短链脂肪酸能降低肠道内环境, 同时可 直接获得较高含水量, 软化粪便, 增加人体排便次数, 防 止便秘, 甚至起到导江作用, 维持肠道清洁, 预防并减少 胃肠道疾病的发生 [10]。

\section{5. 膳食纤维的检测}

目前, 检测食品中膳食纤维含量的常用方法主要分为 洗涤法和酶重量法两大类。洗涤法操作简单方便, 但在测 定过程中损失了可溶性膳食纤维和部分不溶性膳食纤维, 其含量远远低于食物真正的膳食纤维[14], 因此不能适应 膳食纤维分析的需要。酶-重量法是如今应用最多的方法, 于 20 世纪 80 年代在国外首先发展起来, 现已成为国际分析 化学家协会 (AOAC) 认可和推荐的分析方法, 也是目前 公认的测定膳食纤维含量的准确方法 [15]。结合 AOAC985.29、AOAC991.43、AOAC993.19等美国标准, 国内最新制定GB5009.88-2014《食品中膳食纤维的测定》 代替GB/T5009.88-2008《食品中膳食纤维的测定》, 部分 代替 GB/T22224-2008《食品中膳食纤维的测定酶重量法和 酶重量法-液相色谱法》, 该标准针对测定成本昂贵、过 程繁琐以及持续时间长等缺陷进行了探讨和改进。

而爱尔兰Megazyme公司根据AOAC991.43, AOAC 985.29, AACC 32-07和AACC 32-05方法开发了总腼食纤维 检测试剂盒, 实验原理和步骤与国标GB5009.88-2014基本 相同, 但是该检测试剂盒的主要优势在于直接提供高纯度 的酶, 酶的活力是标准化的。淀粉葡萄糖苷酶中基本没有 
纤维素酶, 而其他常用的酶制剂中常含有这种污染物, 这 将导致溶解和低估 $\beta$-葡聚糖。该试剂盒提供的酶都是稳定 的液体, 可以保存到试剂盒有效期, 并且是直接应用液, 不需要使用前再溶解, 方便高效。因此, 本文选用该试剂 盒测定大麦若叶粉中可溶性和不溶性膳食纤维含量。

\section{1. 实验试剂与设备}

\subsection{1. 实验试剂}

A. Megazyme--膳食纤维总量检测试剂盒MK-TDFR $-200 \mathrm{~A}$ [含耐高温 $\alpha$-淀粉酶（20mL， $\sim 3,000 \mathrm{U} / \mathrm{mL}$ ， (Ceralpha method)； $\sim 10,000 \mathrm{U} / \mathrm{mL}$ on soluble starch)1瓶; 纯化的蛋白酶 $(20 \mathrm{~mL}, 50 \mathrm{mg} / \mathrm{mL}$; 350 tyrosineU/mL)1瓶; 纯化的淀粉葡萄糖苷酶 $(20 \mathrm{~mL}, 3300 \mathrm{U} / \mathrm{mL}$ on soluble Starch)2瓶]

B. 硅藻土MG-CEL-100G;

C. MES/TRIS缓冲液 $(0.05 \mathrm{~mol} / \mathrm{L})$ : 称取 $19.52 \mathrm{~g} 2-(\mathrm{N}-$ 吗 啡啉)乙磺酸(MES)(Sigma,M8250)和 $12.2 \mathrm{~g}$ 三羟基氨 基甲烷(TRIS) (Sigma,T1503), 用1.7L蒸馏水溶解, 用 $6 \mathrm{~mol} / \mathrm{L}$ 氢氧化钠调节 $\mathrm{PH}$ 至 $8.2 \pm 0.1$, 加水稀释到 $2 \mathrm{~L}$ (注意 $24^{\circ} \mathrm{C}$ 时 $\mathrm{PH}$ 值为 $8.2,20^{\circ} \mathrm{C}$ 时 $\mathrm{PH}$ 值为 8.3 , $27-28^{\circ} \mathrm{C}$ 时 $\mathrm{PH}$ 值为 8.1 )

D. 盐酸溶液 $(0.561 \mathrm{~mol} / \mathrm{L})$ : 取 $93.5 \mathrm{~mL} 6 \mathrm{~mol} / \mathrm{L}$ 的盐酸, 加入 $700 \mathrm{~mL}$ 水中, 混匀后用水定容到 $1 \mathrm{~L}$ 。

E. $95 \%$ 乙醇AR (体积比)
$78 \%$ 乙醇AR：取 $821 \mathrm{~mL} 95 \%$ 乙醇置于容量瓶中，用水 稀释到刻度, 混匀。

F. 丙酮 $\mathrm{AR}$

\subsection{2. 实验设备}

水浴恒温振荡器SHZ-88A; 电子天平 $0.1 \mathrm{mg}$ FA2204B; 立式冷冻干燥机12ND; 磁力搅拌器78-1; Eppendorf移液 器 $10-100 \mu \mathrm{l} / 20-200 \mu \mathrm{l} / 0.5-5 \mathrm{ml}$; 双列六孔水浴锅DK-S26; 马弗炉SX2-4-10; 恒温干燥箱GZX-DH202-AO-BS; pH 酸度计 PHS-3C; 循环水式多用真空泵SHB-IIIS; 全自动 凯氏定氮仪SKD-800; 智能数控消化炉SKD-20S2。

$60 \mathrm{ml}$ 砂芯坩埚; $400 \mathrm{ml}$ 烧杯; $600 \mathrm{ml}$ 烧杯; 50ml量筒; $250 \mathrm{ml}$ 抽滤瓶; 干燥器; 封口膜; 一次性移液洗头等。

\section{2. 实验原理}

干燥后的样品经耐高温 $\alpha$-淀粉酶、蛋白酶和淀粉葡萄 糖苷酶酶解消化, 酶解液通过乙醇沉淀、过滤, 乙醇和丙 酩洗涤残渣后干燥、称重, 得到总膳食纤维 (TDF) 残渣; 酶解液通过直接过滤、热水洗涤残渣, 干燥后称重, 得到 不溶性膳食纤维 (IDF) 残渣; 滤液用乙醇沉淀, 过滤、 干燥、称重, 得到可溶性膳食纤维 (SDF) 残渣。TDF、 IDF和SDF的残渣扣除蛋白质、灰分和空白即得TDF、IDF 和SDF含量。

\section{3. 实验步骤}

\subsection{1. 实验准备}

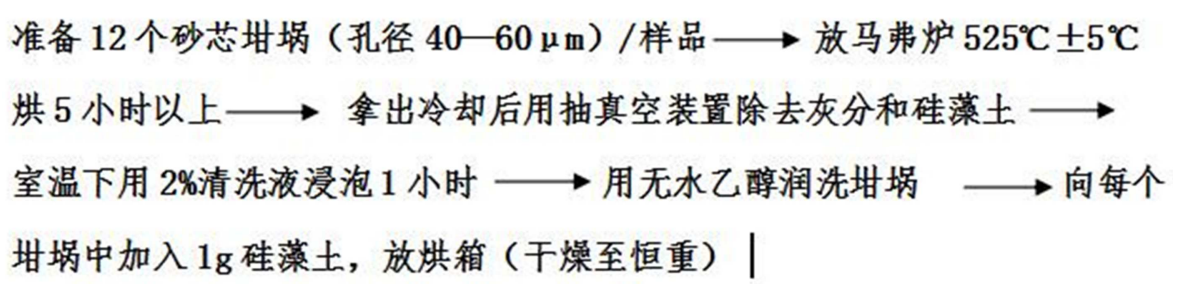

图2 砂芯坩埚前处理流程图/单个样品。

\subsection{2. 大麦若叶粉试样制备}

准确称取四份试样各 $1 \mathrm{~g}$, 质量差小于 $0.005 \mathrm{~g}$, 精确到 $0.1 \mathrm{mg}$, 置于 $400 \mathrm{~mL}$ 高型烧杯中, 同时制备双份空白样, 在每个烧杯中加入 $40 \mathrm{mLpH}$ 值 8.2 的MES-TRIS缓冲液, 磁 力搅拌, 直到试样完全分散到缓冲液中。

\subsection{3. 大麦若叶粉试样酶解}

(1) 耐高温 $\alpha$-淀粉酶酶解：加入 $50 \mu \mathrm{L}$ 耐高温 $\alpha$-淀粉酶溶 液, 加盖铝箔, 置于 $95-100^{\circ} \mathrm{C}$ 恒温振荡水浴锅中持续振摇 （80R）, 当所有烧杯全部放入水浴开始计时, 反应30分 钟。

(2)冷却: 将烧杯取出冷却到 $60^{\circ} \mathrm{C}$, 除去铝箔盖, 若烧 杯内壁和底部发现胶状物, 可用一次性吸管刮下, 用 $10 \mathrm{~mL}$ 蒸馏水冲洗烧杯壁和吸管。
(3)蛋白酶酶解：在每个烧杯中各加入 $100 \mu \mathrm{L}$ 蛋白酶溶 液, 加盖铝箔, 置于 $60^{\circ} \mathrm{C}$ 恒振荡水浴中, 当烧杯内温度达 到 $60^{\circ} \mathrm{C}$ 开始计时, 持续反应 30 分钟。

(4) $\mathrm{pH}$ 值调节: 反应 30 分钟后, 边摚拌边加入 $0.561 \mathrm{~mol} / \mathrm{L}$ 盐酸, 严格控制 $60^{\circ} \mathrm{C}$, 用 $1 \mathrm{~mol} / \mathrm{L}$ 氢氧化钠溶液 或 $1 \mathrm{~mol} / \mathrm{L}$ 盐酸溶液, 控制 $\mathrm{PH}$ 值在 $4.5 \pm 0.2$ 。

(5)淀粉葡萄糖苷酶酶解: 在上述溶液中边搅拌边加入 $200 \mu \mathrm{L}$ 淀粉葡萄糖苷酶, 加盖铝䈃, 置于 $60^{\circ} \mathrm{C}$ 恒温振荡水 浴中, 持续振摇 $(80 \mathrm{R})$, 当烧杯内温度达到 $60^{\circ} \mathrm{C}$ 开始计 时, 反应 30 分钟。

\subsection{4. 大麦若叶粉中IDF与 SDF含量测定}

\section{i. 不溶性膳食纤维测定}

(1)过滤洗涤: 将6份试样酶解液全部转移到坩埚中过 滤, 残渣用 $10 \mathrm{~mL}$ 预热到 $70^{\circ} \mathrm{C}$ 的蒸馏水洗涤两次, 合并过 
滤液, 转移至另一 $600 \mathrm{~mL}$ 高脚烧杯中, 备测可溶性膳食纤 维。残渣分别用 $10 \mathrm{~mL} 95 \%$ 乙醇和丙酮洗涤两次, 抽滤去除 洗涤液, 将坩埚连同残渣在 $103^{\circ} \mathrm{C} \pm 2^{\circ} \mathrm{C}$ 烘干过夜, 将坩埚 置于干燥器中冷却 1 小时, 称重 (包括坩埚、膳食纤维残 渣和硅藻土), 精确至 $0.1 \mathrm{mg}$, 减去坩埚和硅藻土的干重, 计算残渣质量。

(2)蛋白质和灰分测定: 称完质量的残渣和硅藻土的混 合物共 6 份, 取其中 2 份样品残渣和 1 份空白残渣用凯氏定 氮法, 以 $\mathrm{N} \times 6.25$ 为换算系数, 计算蛋白质质量。另 2 份试 样残渣和 1 份样品残渣在 $525^{\circ} \mathrm{C} \pm 5^{\circ} \mathrm{C}$ 灰化 5 小时, 于干燥器 中冷却, 精确称量坩埚总量 (精确至 $0.1 \mathrm{mg}$ ) 减去坩埚和 硅藻土干重, 计算灰分质量。

\section{ii. 可溶性膳食纤维测定}

(1)计算滤液体积: 将不可溶性膳食纤维过滤后的滤液 收集到 $600 \mathrm{~mL}$ 的高型烧杯中，通过烧杯+滤液总重扣除烧 杯质量的方法估算滤液的体积。

(2)沉淀：往滤液中边搅拌边缓慢加入4倍体积预热 $60^{\circ} \mathrm{C}$ 的 $95 \%$ 乙醇，结束后室温下沉淀 1 小时。

(3)过滤：另取6个5.3.1.中前处理好的砂芯坩埚, 用 $15 \mathrm{~mL} 78 \%$ 乙醇将硅藻土润湿, 并用真空溶剂过滤装置在抽 真空条件下, 使硅藻土平铺于坩埚中。将样品酶解液缓慢 转移至对应的坩埚中, 抽滤。用 $78 \%$ 乙醇冲洗将所有残渣 转至坩埚中。

(4)洗涤：分别用 $15 \mathrm{~mL} 78 \%$ 乙醇、 $15 \mathrm{~mL} 95 \%$ 乙醇和 $15 \mathrm{~mL}$ 丙酮洗涤残渣各两次, 抽滤去除洗涤液后, 将坩埚 连同残渣在 $103^{\circ} \mathrm{C} \pm 2^{\circ} \mathrm{C}$ 烘干过夜。将坩埚置于干燥器中冷 却 1 小时, 称重（包括坩埚、膳食纤维残渣和硅藻土）, 精确至 $0.1 \mathrm{mg}$ 。减去坩埚和硅藻土的干重, 计算残渣质量。 方法。

(5)蛋白质和灰分测定: 同5.3.4.1.中蛋白质和灰分测定

\subsection{5. 计算}

实验结果计算公式可参照GB5009.88-2014《食品中膳 食纤维的测定》6.4分析结果的表述内容。

\section{4. 数据分析与处理}

以五种大麦种子种植后制备的大麦若叶粉为样品, 采用上述试剂盒的酶重量法分别测定可溶性与不溶性膳 食纤维含量, 实验过程中记录数据如表 3 , 具体计算结果 见表4。就结果统计, 大麦若叶粉中不可溶性膳食纤维含 量范围为 $1.71 \%-6.69 \%$, 可溶性膳食纤维含量范围为 $0.60 \%-2.26 \%$; 不可溶性膳食纤维两次独立测定结果的 RSD值变化范围为6.02\%-7.97\%, 可溶性膳食纤维两次独 立测定结果的RSD值变化范围为 $0.62 \%-7.74 \%$ 。

表3 测定过程中原始数据值。

\begin{tabular}{llllll}
\hline 样品名 & $\mathbf{m} 1 / \mathbf{g}$ & $\mathbf{m} 2 / \mathbf{g}$ & 灰分/g & 蛋白质/g & $\mathbf{I D F / S D F ( g ) ~}$ \\
\hline 样品空 & 0 & 0 & 0 & 0 & 0 \\
白 & 0 & 0 & 0 & 0 & 0 \\
& 1.0032 & 1.0003 & 0 & 0.0840 & 0.0192 \\
西引3号 & 1.0020 & 0.9990 & 0 & 0.0817 & 0.0171 \\
& 1.0032 & 1.0003 & 0 & 0.0145 & 0.0094 \\
& 1.0020 & 0.9990 & 0 & 0.0152 & 0.0101 \\
& 1.0010 & 1.0008 & 0 & 0.0648 & 0.0270 \\
东辛1号 & 1.0014 & 1.0008 & 0 & 0.0659 & 0.0301 \\
& 1.0010 & 1.0008 & 0 & 0.0112 & 0.0198 \\
& 1.0014 & 1.0008 & 0 & 0.0115 & 0.0197 \\
盐农啤1 & 1.0005 & 1.0026 & 0 & 0.0697 & 0.0568 \\
号 & 1.0027 & 1.0053 & 0 & 0.0670 & 0.0624 \\
& 1.0005 & 1.0026 & 0.0019 & 0.0125 & 0.0226 \\
& 1.0027 & 1.0053 & 0 & 0.0130 & 0.0221 \\
& 1.0014 & 1.0005 & 0 & 0.0788 & 0.0395 \\
苏啤4号 & 1.0054 & 1.0026 & 0 & 0.0804 & 0.0431 \\
& 1.0014 & 1.0005 & 0 & 0.0127 & 0.0060 \\
& 1.0054 & 1.0026 & 0 & 0.0130 & 0.0063 \\
& 1.0013 & 1.0005 & 0 & 0.0725 & 0.0670 \\
苏啤9号 & 1.0002 & 1.0008 & 0 & 0.0715 & 0.0608 \\
& 1.0013 & 1.0005 & 0 & 0.0116 & 0.0114 \\
\hline & 1.0002 & 1.0008 & 0 & 0.0134 & 0.0127 \\
\hline
\end{tabular}

表4 大麦若叶粉中IDF与SDF含量结果。

\begin{tabular}{|c|c|c|c|c|}
\hline 品名 & IDF\%/SDF\% & IDF\%/SDF\%平均值 & IDF\%/SDF\%标准差 & RSD \\
\hline \multirow{3}{*}{ 西引3号 } & $1.91 \%$ & \multirow{2}{*}{$1.81 \%$} & \multirow{2}{*}{$0.14 \%$} & \multirow{2}{*}{$7.97 \%$} \\
\hline & & & & \\
\hline & $0.94 \%$ & $0.97 \%$ & $0.05 \%$ & $5.07 \%$ \\
\hline \multirow{4}{*}{ 东辛1号 } & $2.70 \%$ & \multirow[b]{2}{*}{$2.85 \%$} & \multirow[b]{2}{*}{$0.21 \%$} & \multirow[b]{2}{*}{$7.51 \%$} \\
\hline & $3.00 \%$ & & & \\
\hline & $1.98 \%$ & \multirow{2}{*}{$1.97 \%$} & \multirow{2}{*}{$0.01 \%$} & \multirow{2}{*}{$0.62 \%$} \\
\hline & $1.96 \%$ & & & \\
\hline \multirow{4}{*}{ 盐农啤1号 } & $5.67 \%$ & \multirow{2}{*}{$5.94 \%$} & \multirow{2}{*}{$0.39 \%$} & \multirow{2}{*}{$6.51 \%$} \\
\hline & $6.21 \%$ & & & \\
\hline & $2.26 \%$ & \multirow{2}{*}{$2.23 \%$} & \multirow{2}{*}{$0.04 \%$} & \multirow{2}{*}{$1.74 \%$} \\
\hline & $2.20 \%$ & & & \\
\hline \multirow{3}{*}{ 苏啤4号 } & $3.94 \%$ & \multirow{2}{*}{$4.12 \%$} & \multirow[t]{2}{*}{$0.25 \%$} & \multirow{2}{*}{$6.02 \%$} \\
\hline & $4.29 \%$ & & & \\
\hline & $0.60 \%$ & $0.61 \%$ & $0.01 \%$ & $2.88 \%$ \\
\hline 苏啤9号 & $6.69 \%$ & $6.38 \%$ & $0.43 \%$ & $6.75 \%$ \\
\hline
\end{tabular}




\begin{tabular}{lllll}
\hline 品名 & IDF\%/SDF\% & IDF\%/SDF\%平均值 & IDF\%/SDF\%标准差 & RSD \\
& $6.08 \%$ & & & $7.74 \%$ \\
& $1.14 \%$ & $1.20 \%$ & $0.09 \%$ & \\
\hline
\end{tabular}

\section{6. 结论与展望}

（1）采用该方法测定大麦若叶粉中可溶性与不溶性 膳食纤维含量过程简单, 易操作, 可提高实验效率。

(2) 检测试剂盒直接提供高纯度、稳定的酶液, 减 少酶制剂中常含有的污染物, 在试剂盒有效期内, 直接取 用酶液，方便高效。

（3）在条件允许的情况下, 还需多次平行同一实验, 结合回收率等因素综合确认测得膳食纤维含量数据的精 密度和可靠性。

(4) 本文中设计的实验方案和测定结果为学者们进 一步研究大麦若叶粉的营养功效和生理功能提供参考依 据, 尤其是针对膳食纤维这一营养素特性。此外, 通过相 关的实验研究, 希望能有效提高该类产品在国内市场的关 注度和认可度, 也能进一步推动其在食品中的各项研发应 用。

\section{致谢}

感谢上海交通大学农业与生物学院食品科学与工程 系的隋中泉老师, 在我研读农业推广硕士（食品加工与安 全方向）期间, 提供我实验的机会和条件, 并给予我悉心 的指导和建议。

\section{参考文献}

[1] 张辉, 乔勇进, 戚文元. 大麦苗总黄酮提取工艺对的优化 $[\mathrm{J}]$. 食品与发酵工业，2013，39(8):238-244。

[2] 王炜, 刘春泉, 刘春菊等.大麦若叶苗粉研究进展 [J].食品 工业科技，2017，38(3):395-398。
[3] 段琼辉, 李永, 葛竹兴等.大麦苗粉营养成分分析及评价 [J]. 现代中药研究与实践, 2014, 28(3):55-57。

[4] 王永辉, 李培兵, 李天等.麦苗中氨基酸测定与评价 $[\mathrm{J}]$.氨基 酸和生物资源，2011，33(1):14-15。

[5] 惠秋沙, 李天否.大麦草中微量元素的测定及微量元素营养 分析[J].中国实用医药， 2010，5(25):15-17。

[6] Wild S, Roglic G, Green A, etal.Global prevalence of diabetes:estimates for the year 2000 and projections for 2030 [J]. Diabetes Care, 2004, 27( 5) :1047-1053.

[7] 张辉, 张娜娜, 马丽等.响应面法优化大麦苗叶绿素提取工 艺 $[J]$.食品科学，2014，35(2):75-80。

[8] Hipsley EH.Dietary fiber and pregnancy Toxaemia [J]. British Medical Journal, 1953, 2(4833): 420.

[9] Dai F J, Chau CF.Classification and regulatory perspectives of dietary fiber[J]. Journal of Food and Drug Analysis, 2016, 25(1) : 1-6.

[10] 朱静, 蒋金金金.膳食纤维生理功能的研究进展 [J]. 现代食品, 2016，18(2):4-6。

[11] 吴洪斌, 王永刚, 郑刚等.膳食纤维生理功能研究进展 [J]. 中国酿造，2012，31(3):13-14。

[12] 麦紫欣, 关东华, 林敏霞等.膳食纤维降血脂作用及其机制 的研究进展 $[\mathrm{J}]$.广东微量元素科学, 2011, 18(1):13-15。

[13] 权美平, 侯云云.膳食纤维的生理保健功能及其提取工艺的 研究进展 $[\mathrm{J}]$.保鲜与加工, 2013，13(1):49-51。

[14] 李建文, 杨月欣. 熷食纤维定义及分析方法研究进展 $[\mathrm{J}]$. 食 品科学, 2007(2):350-355。

[15] Malkki Y.Trends in dietary fiber research and development:areview[J].ActaAlimentaria, 2004(33):39-62. 